\title{
Choi-Davis-Jensen Inequalities in Semifinite von Neumann Algebras
}

\author{
Turdebek N. Bekjan, ${ }^{1}$ Kordan N. Ospanov, ${ }^{2}$ and Asilbek Zulkhazhav ${ }^{2}$ \\ ${ }^{1}$ College of Mathematics and Systems Science, Xinjiang University, Urumqi 830046, China \\ ${ }^{2}$ L.N. Gumilyov Eurasian National University, Astana 010008, Kazakhstan \\ Correspondence should be addressed to Turdebek N. Bekjan; bekjant@yahoo.com
}

Received 4 July 2015; Accepted 19 August 2015

Academic Editor: Quanhua Xu

Copyright (c) 2015 Turdebek N. Bekjan et al. This is an open access article distributed under the Creative Commons Attribution License, which permits unrestricted use, distribution, and reproduction in any medium, provided the original work is properly cited.

We prove the Choi-Davis-Jensen type submajorization inequalities on semifinite von Neumann algebras for concave functions and convex functions.

\section{Introduction}

Let $A$ and $\mathscr{B}$ be $C^{*}$-algebras, and let $\Phi$ be a linear map between $\mathscr{A}$ and $\mathscr{B}$. It is said to be positive, if for all positive operators $A \in \mathscr{A}$ we have $\Phi(A) \geq 0$. If for all strictly positive operators $A \in \mathscr{A}(A>0)$, it follows that $\Phi(A)$ is strictly positive, then $\Phi$ is said to be strictly positive. $\Phi$ is called unital if $\Phi(1)=1$, where 1 denotes the unities of the algebras.

Davis [1] and Choi [2] showed that if $\Phi$ is a unital positive linear map on $B(\mathbb{H})$ and if $f$ is an operator convex function on an interval $I$, then the so-called Choi-Davis-Jensen inequality

$$
f(\Phi(A)) \leq \Phi(f(A))
$$

holds for every self-adjoint operator $A$ on $\mathbb{H}$ whose spectrum is contained in $I$, where $B(\mathbb{H})$ is the $C^{*}$-algebra of all bounded linear operators on Hilbert space $\mathbb{H}$. Khosravi et al. [3] proved that (1) still holds for positive linear map $\Phi: \mathscr{A} \rightarrow B(\mathbb{H})$ with $0<\Phi(1) \leq I$. Antezana et al. [4] obtained the following type of Choi-Davis-Jensen inequality. Let $\mathscr{A}, \mathscr{B}$ be unital $C^{*}$ algebras, $\Phi: A \rightarrow B$ a positive unital linear map, $f$ a convex function defined on an open interval $I$, and $A \in \mathscr{A}$ such that $A$ is self-adjoint and $\sigma(a) \subset I$. If $\mathscr{B}$ is a von Neumann algebra and $f$ is monotone, then $f(\Phi(A)) \preceq \Phi(f(A))$ (spectral preorder). One can find some related results to these topics in $[5-8]$.

In [3], the authors proved the following refinement of the Choi-Davis-Jensen inequality: let $\Phi_{1}, \ldots, \Phi_{n}$ be strictly positive linear maps from a unital $C^{*}$-algebra $\mathscr{A}$ into a unital $C^{*}$-algebra $\mathscr{B}$ and let $\Phi=\sum_{j=1}^{n} \Phi_{j}$ be unital. If $f$ is an operator convex function on an interval $I$, then for every selfadjoint operator $A \in \mathscr{A}$ with spectrum contained in $I$,

$$
\begin{aligned}
& f(\Phi(A)) \leq \sum_{j=1}^{n} \Phi_{j}(1)^{1 / 2} \\
& \quad \cdot f\left(\Phi_{j}(1)^{-1 / 2} \Phi_{j}(A) \Phi_{j}(1)^{-1 / 2}\right) \Phi_{j}(1)^{1 / 2} \\
& \quad \leq \Phi(f(A)) .
\end{aligned}
$$

The purpose of this paper is to extend (2) for measurable operators and for convex functions. Let $\mathcal{N}, \mathscr{M}$ be semifinite von Neumann algebras, $\Phi_{1}, \ldots, \Phi_{n}$ positive linear continuous maps from $L_{0}(\mathscr{N})$ into $L_{0}(\mathscr{M})$ such that $\left.\Phi_{1}\right|_{\mathcal{N}}, \ldots,\left.\Phi_{n}\right|_{\mathcal{N}}$ are positive linear maps from $\mathscr{N}$ into $\mathscr{M}$, and $\Phi=\sum_{j=1}^{n} \Phi_{j}$ unital, and let $x_{k} \in L_{0}(\mathcal{N})^{+}(k=1,2, \ldots, n)$. We will prove

$$
\sum_{k=1}^{n} \Phi_{k}\left(f\left(x_{k}\right)\right) \preccurlyeq f\left(\sum_{k=1}^{n} \Phi_{k}\left(x_{k}\right)\right)
$$

for any concave function $f:[0, \infty) \rightarrow[0, \infty)$ and

$$
g\left(\sum_{k=1}^{n} \Phi_{k}\left(x_{k}\right)\right) \preccurlyeq \sum_{k=1}^{n} \Phi_{k}\left(g\left(x_{k}\right)\right)
$$


for any convex function $g:[0, \infty) \rightarrow[0, \infty)$ with $g(0)=0$, where “ $\leqslant$ ” mains submajorization.

This paper is organized as follows. Section 2 contains some preliminary definitions. In Section 3, we prove the main result and related results.

\section{Preliminaries}

We use standard notions from theory of noncommutative $L_{p}$-spaces. Our main references are $[9,10]$ (see also [9] for more historical references). Throughout the paper, let $\mathscr{M}$ be a semifinite von Neumann algebra acting on a Hilbert space $\mathbb{W}$ with a normal semifinite faithful trace $\tau$. Let $L_{0}(\mathscr{M})$ denote the topological $*$-algebra of measurable operators with respect to $(\mathscr{M}, \tau)$. The topology of $L_{0}(\mathscr{M})$ is determined by the convergence in measure. The trace $\tau$ can be extended to the positive cone $L_{0}^{+}(\mathscr{M})$ of $L_{0}(\mathscr{M})$ :

$$
\tau(x)=\int_{0}^{\infty} \lambda d \tau\left(e_{\lambda}(x)\right)
$$

where $x=\int_{0}^{\infty} \lambda d e_{\lambda}(x)$ is the spectral decomposition of $x$.

For $x \in L_{0}(\mathscr{M})$ we define

$$
\begin{aligned}
& \lambda_{s}(x)=\tau\left(e_{s}^{\perp}(|x|)\right) \quad(s>0), \\
& \mu_{t}(x)=\inf \left\{s>0: \lambda_{s}(x) \leq t\right\} \quad(t>0),
\end{aligned}
$$

where $e_{s}^{\perp}(|x|)=e_{(s, \infty)}(|x|)$ is the spectral projection of $|x|$ associated with the interval $(s, \infty)$. The function $s \mapsto$ $\lambda_{s}(x)$ is called the distribution function of $x$ and $\mu_{t}(x)$ is the generalized s-number of $x$. We will denote simply by $\lambda(x)$ and $\mu(x)$ the functions $s \mapsto \lambda_{s}(x)$ and $t \mapsto \mu_{t}(x)$, respectively. It is easy to check that both are decreasing and continuous from the right on $(0, \infty)$. For further information we refer the reader to [11].

If $x, y \in L_{0}(\mathscr{M})$, then we say that $y$ is submajorised by $x$ (in the sense of Hardy, Littlewood, and Polya) and write $y \leqslant x$ if and only if

$$
\int_{0}^{t} \mu_{s}(y) d s \leq \int_{0}^{t} \mu_{s}(x) d s, \quad \forall t>0 .
$$

We remark that if $\mathscr{M}=\mathbb{M}_{n}$ and $\tau$ is the standard trace, then

$$
\mu_{t}(x)=s_{j}(x), \quad t \in[j-1, j), j=1,2, \ldots
$$

and if $x, y \in \mathscr{M}$, then $y \leqslant x$ is equivalent to

$$
\sum_{j=1}^{k} s_{j}(y) \leq \sum_{j=1}^{k} s_{j}(x), \quad 1 \leq k \leq n .
$$

For further information we refer the reader to [11-13].

Let $x, y$ be self-adjoint elements of $\mathscr{M}$; we say that $x$ spectrally dominates $y$, denoted by $y \preceq x$, if $e_{(\alpha, \infty)}(y)$ is equivalent, in the sense of Murray-von Neumann, to a subprojection of $e_{(\alpha, \infty)}(x)$ for every real number $\alpha$ (see [6]). It is clear that if $x$ spectrally dominates $y$, then $y$ is submajorised by $x$.

\section{Main Results}

Lemma 1. Let $\mathcal{N}$ and $\mathscr{M}$ be semifinite von Neumann algebras. Let $\Phi$ be a positive linear continuous map from $L_{0}(\mathcal{N})$ into $L_{0}(\mathscr{M})$ such that the restriction of $\Phi$ on $\mathcal{N}$ is a unital positive linear map from $\mathcal{N}$ into $\mathscr{M}$.

(i) If $f:[0,+\infty) \rightarrow[0,+\infty)$ is a concave function, then

$$
\Phi(f(x)) \preccurlyeq f(\Phi(x)), \quad \forall x \in L_{0}(\mathcal{N})^{+} .
$$

(ii) If $g:[0,+\infty) \rightarrow[0,+\infty)$ is a convex function with $g(0)=0$, then

$$
g(\Phi(x)) \leqslant \Phi(g(x)), \quad \forall x \in L_{0}(\mathcal{N})^{+} .
$$

Proof. (i) We may assume $f(0)=0$. It implies $f$ is nondecreasing. First assume that $x \in \mathcal{N}^{+}$. We use same method as in the proof of Theorem 2 [4] (see Remark 3.2 in [4]). Let $a>0$. If $\xi \in e_{\{t: f(t) \leq a\}}(\Phi(x))(\mathscr{H}) \cap e_{(a, \infty)}(\Phi(f(x)))(\mathscr{H})$ with $\|\xi\|=1$, then $f(\langle\Phi(x) \xi, \xi\rangle) \leq a$ and $\langle\Phi(f(x)) \xi, \xi\rangle>$ $a$. On the other hand, using Jensen's inequality for the state $\langle\Phi(\cdot) \xi, \xi\rangle$ and nondecreasing concave function $f$, we get $\langle\Phi(f(x)) \xi, \xi\rangle \leq f(\langle\Phi(x) \xi, \xi\rangle) \leq a$. Therefore $e_{\{t: f(t) \leq a\}}(\Phi(x))(\mathscr{H}) \cap e_{(a, \infty)}(\Phi(f(x)))(\mathscr{H})=\{0\}$. Thus $e_{\{t: f(t) \leq a\}}(\Phi(x)) \wedge e_{(a, \infty)}(\Phi(f(x)))=0$ and

$$
\begin{aligned}
e_{(a, \infty)}(\Phi(f(x)))= & e_{(a, \infty)}(\Phi(f(x))) \\
& -e_{\{t: f(t) \leq a\}}(\Phi(x)) \\
& \wedge e_{(a, \infty)}(\Phi(f(x))) \\
\sim & e_{\{t: f(t) \leq a\}}(\Phi(x)) \\
& \vee e_{(a, \infty)}(\Phi(f(x))) \\
& -e_{\{t: f(t) \leq a\}}(\Phi(x)) \\
\leq & 1-e_{\{t: f(t) \leq a\}}(\Phi(x)) \\
= & e_{\{t: f(t)>a\}}(\Phi(x)) \\
= & e_{(a,+\infty)}(f(\Phi(x))) ;
\end{aligned}
$$

that is, $\Phi(f(x)) \preceq f(\Phi(x))$. Hence (10) holds.

Now let $x \in L_{0}(\mathcal{N})^{+}$. For each $m=1,2, \ldots$, observe that $x \wedge m 1 \in \mathscr{M}$, and so, using the first case, it follows that

$$
\Phi(f(x \wedge m 1)) \preccurlyeq f(\Phi(x \wedge m 1)) .
$$

Using the functional calculus and Corollary 1.2 in [13] observe that

$$
\begin{gathered}
x \wedge m 1 \uparrow_{m} x, \\
f(x \wedge m 1) \uparrow_{m} f(x)
\end{gathered}
$$

and so, by continuousness of $\Phi$, it follows that

$$
\begin{gathered}
\Phi(x \wedge m 1) \uparrow_{m} \Phi(x), \\
\Phi(f(x \wedge m 1)) \uparrow_{m} \Phi(f(x)) .
\end{gathered}
$$


Using (vi) of Lemma 2.5 in [11] we obtain that

$$
\begin{gathered}
\int_{0}^{t} \mu_{s}(f(\Phi(x))) d s=\int_{0}^{t} f\left(\mu_{s}(\Phi(x))\right) d s \\
\quad=\lim _{m \rightarrow \infty} \int_{0}^{t} f\left(\mu_{s}(\Phi(x \wedge m 1))\right) d s \\
\quad=\lim _{m \rightarrow \infty} \int_{0}^{t} \mu_{s}(f(\Phi(x \wedge m 1))) d s \\
\geq \lim _{m \rightarrow \infty} \int_{0}^{t} \mu_{s}(\Phi(f(x \wedge m 1))) d s \\
=\int_{0}^{t} \mu_{s}(\Phi(f(x))) d s, \quad \forall t>0 ;
\end{gathered}
$$

that is, (10) holds.

(ii) The proof is similar to the proof of (i).

Theorem 2. Let $\mathcal{N}, \mathscr{M}$ be semifinite von Neumann algebras, $\Phi_{1}, \ldots, \Phi_{n}$ positive linear continuous maps from $L_{0}(\mathcal{N})$ into $L_{0}(\mathscr{M})$ such that the restriction of $\Phi_{k}$ on $\mathcal{N}$ is a positive linear map from $\mathcal{N}$ into $\mathscr{M}(k=1,2, \ldots, n)$, and $\Phi=\sum_{j=1}^{n} \Phi_{j}$ unital.

(i) If $f:[0,+\infty) \rightarrow[0,+\infty)$ is a concave function, then, for $x_{k} \in L_{0}(\mathcal{N})^{+}(k=1,2, \ldots, n)$,

$$
\sum_{k=1}^{n} \Phi_{k}\left(f\left(x_{k}\right)\right) \preceq f\left(\sum_{k=1}^{n} \Phi_{k}\left(x_{k}\right)\right) \text {. }
$$

(ii) If $g:[0,+\infty) \rightarrow[0,+\infty)$ is a convex function with $g(0)=0$, then, for $x_{k} \in L_{0}(\mathcal{N})^{+}(k=1,2, \ldots, n)$,

$$
g\left(\sum_{k=1}^{n} \Phi_{k}\left(x_{k}\right)\right) \preceq \sum_{k=1}^{n} \Phi_{k}\left(g\left(x_{k}\right)\right) \text {. }
$$

Proof. Let $\mathcal{N}_{n}$ be the von Neumann algebra:

$$
\begin{aligned}
& \mathscr{N}_{n}=\left\{\left(\begin{array}{cccc}
x_{1} & 0 & \cdots & 0 \\
0 & x_{2} & \cdots & 0 \\
& & \ddots & \\
0 & 0 & \cdots & x_{n}
\end{array}\right), x_{k} \in \mathcal{N}, k\right. \\
& =1,2, \ldots, n\}
\end{aligned}
$$

on Hilbert space $\mathscr{H} \oplus \cdots \oplus \mathscr{H}$. Define $\Phi: \mathcal{N}_{n} \rightarrow \mathscr{M}$ by

$$
\Phi\left(\left(\begin{array}{cccc}
x_{1} & 0 & \cdots & 0 \\
0 & x_{2} & \cdots & 0 \\
& & \ddots & \\
0 & 0 & \cdots & x_{n}
\end{array}\right)\right)=\sum_{k=1}^{n} \Phi_{k}\left(x_{k}\right) ;
$$

then $\Phi$ is a unital positive linear map from $\mathcal{N}_{n}$ into $\mathscr{M}$. By Lemma 1, we obtain desired result.
Using Theorem 5.3 in [14] and Theorem 2 we obtain the following.

Proposition 3. Let $\mathcal{N}, M$ be semifinite von Neumann algebras, $\Phi_{1}, \ldots, \Phi_{n}$ positive linear continuous maps from $L_{0}(\mathcal{N})$ into $L_{0}(\mathscr{M})$ such that the restriction of $\Phi_{k}$ on $\mathcal{N}$ is a positive linear map from $\mathcal{N}$ into $\mathscr{M}(k=1,2, \ldots, n)$, and $\Phi=\sum_{j=1}^{n} \Phi_{j}$ unital.

(i) If $f:[0,+\infty) \rightarrow[0,+\infty)$ is a concave function, then, for $x_{k} \in \mathcal{N}^{+}(k=1,2, \ldots, n)$,

$$
\sum_{k=1}^{n} \Phi_{k}\left(f\left(x_{k}\right)\right) \preccurlyeq f\left(\sum_{k=1}^{n} \Phi_{k}\left(x_{k}\right)\right) \preccurlyeq \sum_{k=1}^{n} f\left(\Phi_{k}\left(x_{k}\right)\right) .
$$

(ii) If $g:[0,+\infty) \rightarrow[0,+\infty)$ is a convex function with $g(0)=0$, then, for $x_{k} \in \mathcal{N}^{+}(k=1,2, \ldots, n)$,

$$
\sum_{k=1}^{n} g\left(\Phi_{k}\left(x_{k}\right)\right) \preccurlyeq g\left(\sum_{k=1}^{n} \Phi_{k}\left(x_{k}\right)\right) \preccurlyeq \sum_{k=1}^{n} \Phi_{k}\left(g\left(x_{k}\right)\right) \text {. }
$$

Proposition 4. Let $\mathcal{N}, \mathscr{M}$ be semifinite von Neumann algebras and $\Phi_{1}, \ldots, \Phi_{n}$ positive linear continuous maps from $L_{0}(\mathcal{N})$ into $L_{0}(\mathscr{M})$ such that the restriction of $\Phi_{k}$ on $\mathcal{N}$ is a positive linear map from $\mathcal{N}$ into $\mathscr{M}(k=1,2, \ldots, n)$. Suppose $\Phi=$ $\sum_{j=1}^{n} \Phi_{j}$ is unital trace-preserving positive linear map from $\mathscr{M}$ into $\mathscr{M}$. If $g:[0,+\infty) \rightarrow[0,+\infty)$ is a convex function with $g(0)=0$, then

$$
\begin{aligned}
\sum_{k=1}^{n} g\left(\Phi_{k}(x)\right) & \preccurlyeq g\left(\sum_{k=1}^{n} \Phi_{k}(x)\right) \preccurlyeq \sum_{k=1}^{n} \Phi_{k}(g(x)) \\
& \preccurlyeq g(x), \quad \forall x \in \mathscr{M}^{+} .
\end{aligned}
$$

Proof. By Corollary 2.9 in [15] we have that $\sum_{j=1}^{n} \Phi_{j}$ is a tracepreserving positive contraction. Using Theorem 5.3 in [14], Lemma 3.1 in [16] (it is also holds for the semifinite case), and Theorem 2 we obtain the desired result.

Corollary 5. Let $a_{k} \in \mathscr{M}(k=1,2, \ldots, n)$ and $\sum_{k=1}^{n} a_{k}^{*} a_{k}=1$.

(i) If $f:[0,+\infty) \rightarrow[0,+\infty)$ is a concave function, then, for $x_{k} \in L_{0}(\mathscr{M})^{+}(k=1,2, \ldots, n)$,

$\sum_{k=1}^{n} a_{k}^{*} f\left(x_{k}\right) a_{k} \preccurlyeq f\left(\sum_{k=1}^{n} a_{k}^{*} x_{k} a_{k}\right) \preccurlyeq \sum_{k=1}^{n} f\left(a_{k}^{*} x_{k} a_{k}\right)$.

(ii) If $g:[0,+\infty) \rightarrow[0,+\infty)$ is a convex function with $g(0)=0$, then

$$
\begin{aligned}
\sum_{k=1}^{n} g\left(a_{k}^{*} x a_{k}\right) & \preccurlyeq g\left(\sum_{k=1}^{n} a_{k}^{*} x a_{k}\right) \preccurlyeq \sum_{k=1}^{n} a_{k}^{*} g(x) a_{k} \\
& \preccurlyeq g(x), \quad \forall x \in L_{0}(\mathscr{M})^{+} .
\end{aligned}
$$

Let $\mathbb{M}_{n}$ be von Neumann algebra of $n \times n$ complex matrices, and let $P_{1}, P_{2}, \ldots, P_{r}$ be a family of mutually orthogonal 
projections in $\mathbb{C}^{n}$ such that $\oplus_{j=1}^{r} P_{j}=I$, where $I$ is unit matrix in $\mathbb{M}_{n}$. Then the operation of taking $A$ to $\mathscr{C}(A)=\sum_{j=1}^{r} P_{j} A P_{j}$ is called a pinching of $A$. The pinching $\mathscr{C}: \mathbb{M}_{n} \rightarrow \mathbb{M}_{n}$ is a trace-preserving positive map (see $[17,18]$ ).

Proposition 6. Let $\mathscr{C}: \mathbb{M}_{n} \rightarrow \mathbb{M}_{n}$ be a pinching.

(i) If $f:[0,+\infty) \rightarrow[0,+\infty)$ is a concave function, then

$$
\mathscr{C}(f(A)) \preccurlyeq f(\mathscr{C}(A)), \quad \forall A \in \mathbb{M}_{n}^{+} .
$$

(ii) If $g:[0,+\infty) \rightarrow[0,+\infty)$ is a convex function with $g(0)=0$, then

$$
g(\mathscr{C}(A)) \preccurlyeq \mathscr{C}(g(A)) \leqslant g(A), \quad \forall A \in \mathbb{M}_{n}^{+} .
$$

Let $x=\left(x_{k}\right)_{k \geq 1}$ be a sequence in $L_{0}(\mathscr{M})$. Define

$$
\operatorname{diag}\left(x_{k}\right)=\left(\begin{array}{ccccc}
x_{1} & 0 & \cdots & 0 & \cdots \\
0 & x_{2} & \cdots & 0 & \cdots \\
& & \ddots & & \\
0 & 0 & \cdots & x_{k} & \cdots \\
& & & & \ddots
\end{array}\right) .
$$

Proposition 7. Let $x=\left(x_{k}\right)_{k \geq 1}$ be a sequence in $L_{0}(\mathscr{M})$.

(i) If $f:[0,+\infty) \rightarrow[0,+\infty)$, where $f(\sqrt{t})$ is a concave function, then

$$
\begin{array}{r}
\left\|f\left(\left(\sum_{k \geq 1}\left|x_{k}\right|^{2}\right)^{1 / 2}\right)\right\|_{p} \leq\left(\sum_{k \geq 1}\left\|f\left(\left|x_{k}\right|\right)\right\|^{p}\right)^{1 / p}, \\
\forall 0<p \leq 1 .
\end{array}
$$

(ii) If $g:[0,+\infty) \rightarrow[0,+\infty)$, where $g(\sqrt{t})$ is a convex function with $g(0)=0$, then

$$
\left(\sum_{k \geq 1}\left\|g\left(\left|x_{k}\right|\right)\right\|^{p}\right)^{1 / p} \leq\left\|g\left(\left(\sum_{k \geq 1}\left|x_{k}\right|^{2}\right)^{1 / 2}\right)\right\|_{p},
$$

$$
\forall 1 \leq p<\infty .
$$

Proof. (i) Since

$$
\begin{aligned}
\left(\begin{array}{ccc}
\left|x_{1}^{*}\right|^{2} & 0 & \cdots \\
0 & \left|x_{2}^{*}\right|^{2} & \cdots \\
\vdots & \vdots & \ddots
\end{array}\right) \\
=\mathscr{C}\left(\left(\begin{array}{ccc}
\left|x_{1}^{*}\right|^{2} & x_{1} x_{2}^{*} & \cdots \\
x_{2} x_{1}^{*} & \left|x_{2}^{*}\right|^{2} & \cdots \\
\vdots & \vdots & \ddots
\end{array}\right)\right) \\
=\mathscr{C}\left(\left(\begin{array}{ccc}
x_{1} & 0 & \cdots \\
x_{2} & 0 & \cdots \\
\vdots & \vdots & \ddots
\end{array}\right)\left(\begin{array}{ccc}
x_{1}^{*} & x_{2}^{*} & \cdots \\
0 & 0 & \cdots \\
\vdots & \vdots & \ddots
\end{array}\right)\right)
\end{aligned}
$$

and $f(\sqrt{t})^{p}$ is concave, by Lemma 1 , we get

$$
\begin{gathered}
\mathscr{C}\left(f^{p}\left(\left|\left(\begin{array}{ccc}
x_{1}^{*} & x_{2}^{*} & \cdots \\
0 & 0 & \cdots \\
\vdots & \vdots & \ddots
\end{array}\right)\right|\right)\right) \\
\leqslant\left(\begin{array}{ccc}
f\left(\left|x_{1}^{*}\right|\right)^{p} & 0 & \cdots \\
0 & f\left(\left|x_{2}^{*}\right|\right)^{p} & \cdots \\
\vdots & \vdots & \ddots
\end{array}\right) .
\end{gathered}
$$

Hence

$$
\begin{aligned}
& \left\|f\left(\left(\sum_{k \geq 1}\left|x_{k}\right|^{2}\right)^{1 / 2}\right)\right\|_{p}^{p} \\
& =\tau\left(f^{p}\left(\left|\left(\begin{array}{ccc}
x_{1} & 0 & \cdots \\
x_{2} & 0 & \cdots \\
\vdots & \vdots & \ddots
\end{array}\right)\right|\right)\right) \\
& =\tau\left(f^{p}\left(\left|\left(\begin{array}{ccc}
x_{1}^{*} & x_{2}^{*} & \cdots \\
0 & 0 & \cdots \\
\vdots & \vdots & \ddots
\end{array}\right)\right|\right)\right) \\
& =\tau\left(\mathscr{C}\left(f^{p}\left(\left|\left(\begin{array}{ccc}
x_{1}^{*} & x_{2}^{*} & \cdots \\
0 & 0 & \cdots \\
\vdots & \vdots & \ddots
\end{array}\right)\right|\right)\right)\right) \\
& \leq \tau\left(\left(\begin{array}{ccc}
f\left(\left|x_{1}^{*}\right|\right)^{p} & 0 & \cdots \\
0 & f\left(\left|x_{2}^{*}\right|\right)^{p} & \ldots \\
\vdots & \vdots & \ddots
\end{array}\right)\right) \\
& =\sum_{k \geq 1}\left\|f\left(\left|x_{k}\right|\right)\right\|^{p} .
\end{aligned}
$$

Using the same arguments, we can prove (ii).

\section{Conflict of Interests}

The authors declare that there is no conflict of interests regarding the publication of this paper.

\section{Acknowledgments}

Turdebek N. Bekjan is partially supported by NSFC Grant no. 11371304. Kordan N. Ospanov is partially supported by Project 3606/GF4 of Science Committee of Ministry of Education and Science of the Republic of Kazakhstan. 


\section{References}

[1] C. Davis, "A Schwarz inequality for convex operator functions," Proceedings of the American Mathematical Society, vol. 8, pp. 4244, 1957.

[2] M. D. Choi, "A Schwarz inequality for positive linear maps on $C^{*}$-algebras," Illinois Journal of Mathematics, vol. 18, pp. 565$574,1974$.

[3] M. Khosravi, J. S. Aujla, S. S. Dragomir, and M. S. Moslehian, "Refinements of Choi-Davis-Jensen's inequality," Bulletin of Mathematical Analysis and Applications, vol. 3, no. 2, pp. 127133, 2011.

[4] J. Antezana, P. Massey, and D. Stojanoff, “Jensen's inequality for spectral order and submajorization," Journal of Mathematical Analysis and Applications, vol. 331, no. 1, pp. 297-307, 2007.

[5] T. Ando, Topics on Operator Inequalities, Hokkaido University, Sapporo, Japan, 1978.

[6] L. G. Brown and H. Kosaki, "Jensen's inequality in semi-finite von Neumann algebras," Journal of Operator Theory, vol. 23, no. 1, pp. 3-19, 1990.

[7] F. Hansen and G. K. Pedersen, "Jensen's operator inequality," The Bulletin of the London Mathematical Society, vol. 35, no. 4, pp. 553-564, 2003.

[8] F. Hansen and G. K. Pedersen, "Jensen's inequality for operators and Lowner's theorem," Mathematische Annalen, vol. 258, no. 3, pp. 229-241, 1982.

[9] G. Pisier and Q. Xu, "Noncommutative $L_{p}$-spaces," in Handbook of the Geometry of Banach Spaces, vol. 2, pp. 1459-1517, 2003.

[10] Q. Xu, Noncommutative $\mathrm{L}_{p}$-Spaces and Martingale Inequalities, 2007.

[11] T. Fack and H. Kosaki, "Generalized $s$-numbers of $\tau$-measure operators," Pacific Journal of Mathematics, vol. 123, no. 2, pp. 269-300, 1986.

[12] P. G. Dodds, T. K. Dodds, and B. de Pagter, "Noncommutative Banach function spaces," Mathematische Zeitschrift, vol. 201, no. 4, pp. 583-597, 1989.

[13] P. G. Dodds, T. K. Dodds, and B. de Pager, "Noncommutative Köthe duality," Transactions of the American Mathematical Society, vol. 339, pp. 717-750, 1993.

[14] P. G. Dodds and F. A. Sukochev, "Submajorisation inequalities for convex and concave functions of sums of measurable operators," Positivity., vol. 13, no. 1, pp. 107-124, 2009.

[15] V. Paulsen, Completely Bounded Maps and Operator Algebras, vol. 78 of Cambridge Studies in Advanced Mathematics, Cambridge University Press, 2002.

[16] T. N. Bekjan and M. Raikhan, "An Hadamard-type inequality," Linear Algebra and its Applications, vol. 443, pp. 228-234, 2014.

[17] R. Bhatia, M. D. Choi, and C. Davis, "Comparing a matrix to its off-diagonal part," Operator Theory: Advances and Applications, vol. 40, pp. 151-163, 1989.

[18] J.-C. Bourin and M. Uchiyama, "A matrix subadditivity inequality for $f(A+B)$ and $f(A)+f(B)$," Linear Algebra and its Applications, vol. 423, no. 2-3, pp. 512-518, 2007. 


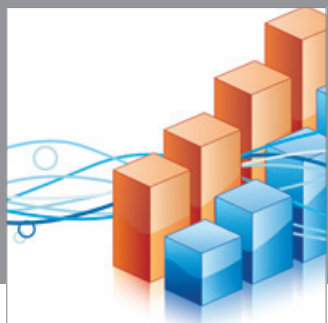

Advances in

Operations Research

mansans

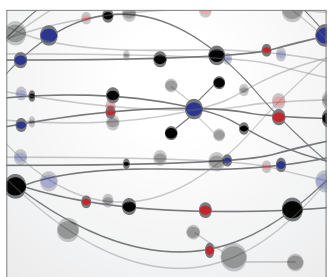

The Scientific World Journal
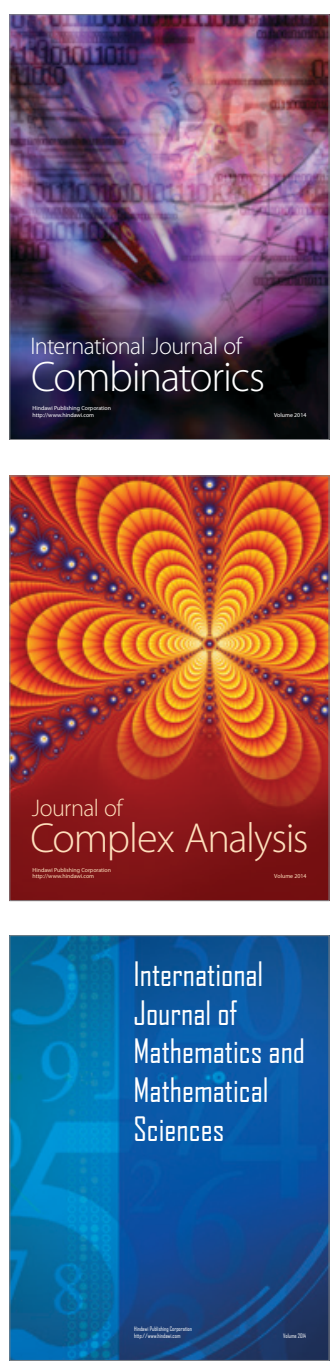
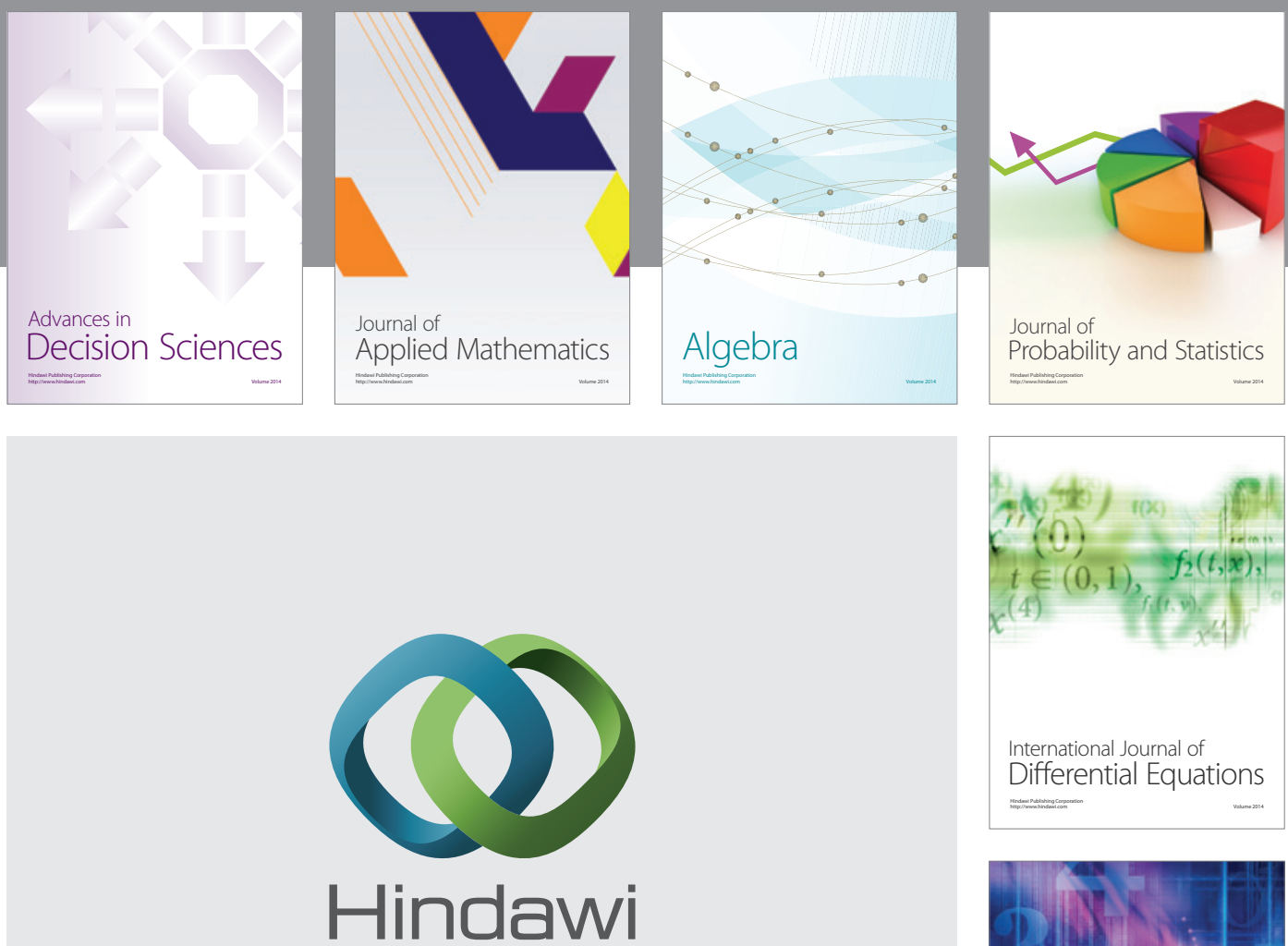

Submit your manuscripts at http://www.hindawi.com
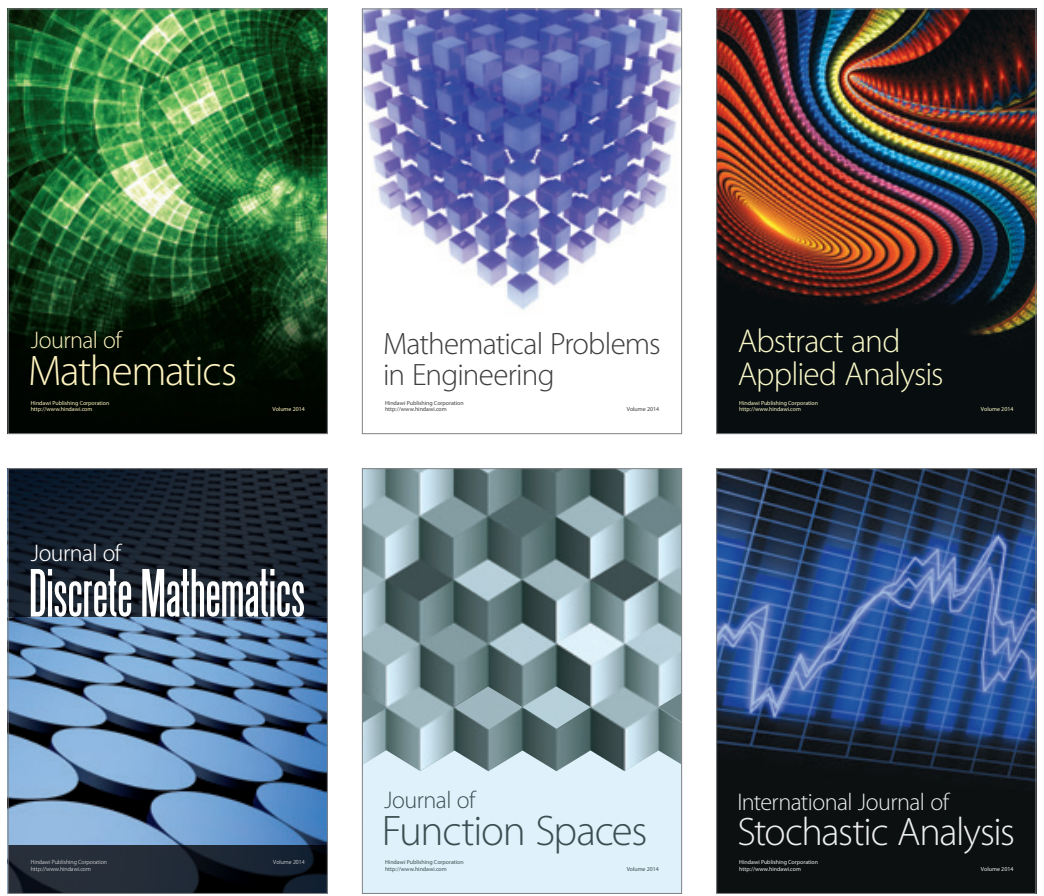

Journal of

Function Spaces

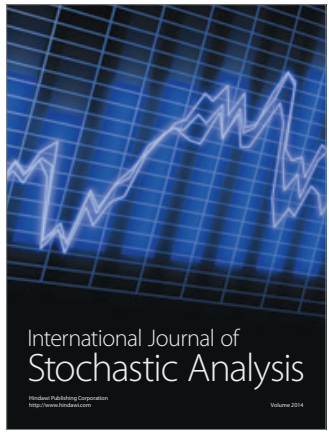

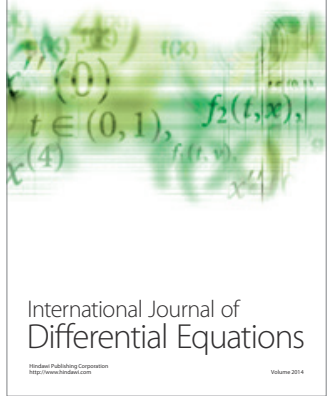
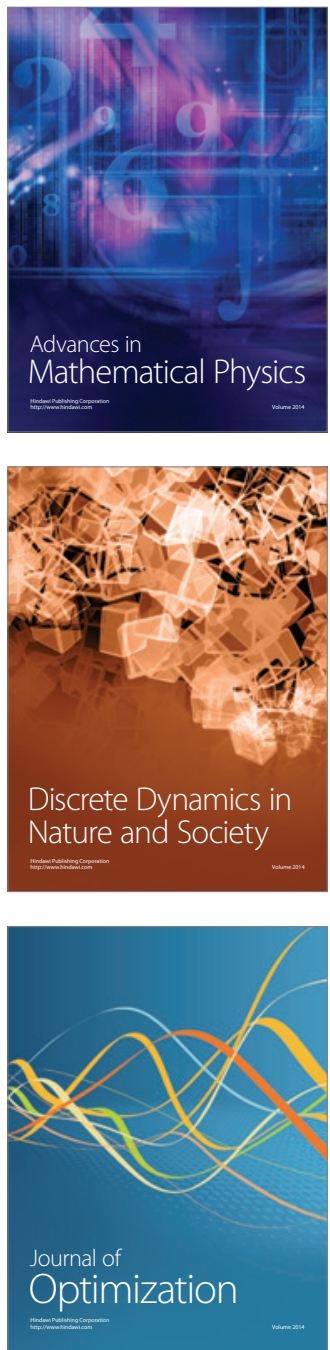